\title{
Proximal Femoral Nailing: Technical Difficulties and Results in Trochanteric Fractures
}

\author{
Janardhana Aithala P, Sharath Rao
}

Department of Orthopedics, Kasturba Medical College, Manipal, India.

Email: janardanaaithala@yahoo.com

Received June $5^{\text {th }}, 2013$; revised July $8^{\text {th }}, 2013$; accepted July $22^{\text {nd }}, 2013$

Copyright (C) 2013 Janardhana Aithala P, Sharath Rao. This is an open access article distributed under the Creative Commons Attribution License, which permits unrestricted use, distribution, and reproduction in any medium, provided the original work is properly cited.

\begin{abstract}
Background: Proximal femoral nailing in communited intertrochanteric fractures is increasingly becoming popular in view of superior biomechanics and prevention of varus collapse associated with Dynamic hip screw. However, technical difficulties and implant related complications have been described with this technique, thus we need more studies to address these issues. Our study aims to understand technical difficulties involved in proximal femoral nailing, and specifically analyses neck shaft angle at follow-up indicating varus collapse and also to compare results of stable and unstable fractures. Materials and Methods: In this study, patients who presented to the Orthopedic Unit of Dr. TMA Pai Hospital (An associated hospital of Manipal University, Manipal) with trochanteric fractures included and treated with proximal femoral nailing. The technical difficulties involved with surgical procedure and techniques adapted to overcome such difficulties were recorded. All patients were followed up for a period of 2 years and final outcome assessment included the number of shortening, neck shaft angle and harris hip score. Results: 41 patients (mean age 71) who underwent proximal femoral nailing from January 2004 to December 2009 were included in the study, 38 patients completed 2-year follow-up. The technical difficulties we faced were divided into 3 categories, difficulties in securing entry point and guide wire placement especially when greater trochanter and piriform fossa were gathered, reduction was lost while passing nail, and finally difficulties faced during placement of hip screws. In all except one, neck shaft angle of more than 130 degrees was achieved, and this was also maintained in the final follow-up (Mean 131.9 degrees). All fractures were united, with mean shortening of $2 \mathrm{~mm}$. Conclusions: Although PFN is technically required, with a proper technique PFN gives excellent clinical results with almost negligible varus collapse even in unstable trochanteric fractures. Regarding the techniques, reaming the proximal part of femur adequately and observing the nail passage with image carefully are important in placing the nail correctly, while, placement of lag screw in the inferior part of neck in anterior posterior projection and central in lateral projection reduces risk of implant failure.
\end{abstract}

Keywords: Trochanteric Fractures; Proximal Femoral Nailing; Varus Collapse

\section{Introduction}

Intertrochanteric fractures are one of the commonest fractures in elderly people $[1,2]$. With the increase in life expectancy, we are seeing more and more such fractures in our practice today [3]. Following the intertrochanteric fractures as it affects elderly people, quality of life will be poor unless fractures are stabilized and mobilized early.

With the aim of mobilizing these patients early from the bed, surgical stabilization of these fractures is favored $[3,4]$ and many fixation devices are developed. However, sliding hip screw device with a slide plate remains the

${ }^{*}$ Conflict of interest: None. gold standard [3,5]. Although these devices produce acceptable reduction and healing in stable intertrochanteric fractures, in unstable fractures, the results are unsatisfactory [5-7]. Only about $50 \%$ can be expected to reach the pre-injury activity status. Although union is not a big problem in these patients, a significant number of patients will be confined to home and have significant shortening due to excessive collapse in unstable trochanteric fractures [6-10]. To overcome these difficulties, a biomechanically more stable, intramedullary device has been tried. Theoretically, there is an improved biomechanical environment with an intramedullary device, with a shorter lever arm, which provides more load shar- 
ing and allows less collapse for a stable medial configuration [7]. Also with limited access approach, one can reduce the blood loss, soft tissue trauma and infection. Earlier results with Gamma nail did not produce any significant benefit above sliding hip screw while there were additional complications in perioperative and postoperative proximal femoral fractures and also technical difficulties while inserting the gamma nail $[7,9,11,12]$. Many modifications were made, out of which it included proximal femoral nail with slightly reduced proximal diameter of nail and two screw systems gained popularity [9,13-15]. However, till today, in view of complications like $\mathrm{Z}$ effect, proximal femoral fracture and technical difficulties, people still prefer DHS as the gold standard as there are contradictory reports regarding the superiority of one over the other. Various studies on intramedullary devices have shown that there are complications with nail to make screw cut out, proximal femoral fracture, higher reoperation rates, wound infection [8,9,13,15-18]. Also, none of these studies have studied or described how neck shaft angle is maintained in patients treated with PFN, thus highlighting the superiority of PFN over DHS in preventing varus collapse.

Following difficulties with Dynamic hip screw fixation, especially in unstable trochanteric fractures, the authors have started using proximal femoral nail in trochanteric fractures since January 2004. This study aims to understand the technical difficulties which are the main problem in using these nails and finding a solution to these problems. The study also aims to analyze the overall results of proximal femoral nailing and look at the incidence of varus collapse (loss of neck shaft angle) specifically following union, and to see if there is any difference between stable and unstable fractures with regard to neck shaft angle.

\section{Materials and Methods}

All patients with pertrochanteric fractures from January 2004 to December 2009 were included in the study and treated with proximal femoral nailing, using either Pitkar or Inor make. All the patients gave the informed consent for inclusion into study and the study was authorized by the local ethical committee (UEC/04/13-14, Manipal university ethics committee) and was performed in accordance with the Ethical standards of the 1964 Declaration of Helsinki as revised in 2000 (WMA declaration of Helsinki-Ethical principles for medical research,

www.wma.net). All patients were evaluated at the end of 2 years. Fractures were classified using modified Evan's classification $[19,20]$ as follows:

Stable:

Type 1: undisplaced, 2 part fractures.

Type 2: displaced but can be reduced to a stable posi- tion.

\section{Unstable:}

Type 3: with communition of greater trochanter and loss of posterolateral stability.

Type 4: with communition around lesser trochanter and loss of medial stability.

Type 5: with loss of both medial and posterolateral stability.

$\mathrm{R}$ : with reverse obliquity.

Assessment of patient's preoperative assessment included, fracture classification, age, preoperative mobility status, Harris hip score [21] and co moribund conditions.

Surgical technique included closed reduction (if there was large spiral subtrochanteric fragment, minimal access reduction was done using a bone holding forceps) and closed intramedullary nailing with proximal femoral nail. Surgeon was experienced in passing second generation intramedullary nails for femoral shaft fractures as well as RT nails for complex femoral fractures. Despite this, the time duration and complications in first 10 cases were separately analyzed, as during these initial cases we made several technical adjustments for the technique and hence our surgical time and difficulty with which we passed the nail became less in the subsequent cases. The intraoperative characteristics studied were duration of surgery, blood loss, technical difficulties during surgery along with complications. For understanding the techniques of PFN better, the technical details were classified into:

1) Entry point and guide wire placement problems: Problems related to reduction; Problems related to entry point; Problems related to guide wire passage.

2) Problems related to passing Nail.

3) Problems related to locking the nail with Lag screw and derotational screws.

Methods used to solve these problems were noted in the operative notes and these data were used for discussion and analysis.

Postoperative characteristics studied are list of complications, postoperative mobilization status, subsequent return to maximum mobility status or death, amount of shortening, Harris hip score at 2 years, and also radiological assessment which included analysis of neck shaft angle and implant status.

\section{Results}

A total of 41 patients were treated with Proximal femoral nail from January 2004 to December 2009. 3 patients expired before 6 months with 2 of them within 1 month, hence 38 patients were available for final follow up. Mean age of patients was 71.39.

As per Evan's classification, there were two type 1 fractures, fourteen type 2 fractures (thus a total of 16 fractures which were considered stable), eight type 3 fractures, eight type 4 fractures, six type 5 fractures and 
two reverse oblique fractures (thus a total of 24 patients with unstable fractures) and finally one patient had a subtrochanteric fracture.

3 patients expired before 6 months and hence excluded from the analysis of results. In the remaining 38 patients, 2 patients need walker, one patient is not able to walk, and 15 patients walk comfortably with the help of a single stick. Remaining 20 patients walk without the stick. In contrast, before the injury, 2 patients were using walker and 7 patients were using single stick. Functional assessment with harris hip score showed almost near return to preinjury score (preinjury score of 71 versus final follow-up mean score of 66). Except 2 patients all fractures showed union within 6 months. Remaining 2 patients, showed union at the end of 9 months. Mean neck shaft angle achieved post reduction was 132.4 degrees (38 patients) and at final follow up was 131.9 degrees (33 patients) with insignificant difference (Mean difference of 0.5 degrees). Limb length discrepancy was assessed in the final follow up and only two patients had shortening of more than $2 \mathrm{~cm}(20 \mathrm{~mm})$, with average shortening of 2 $\mathrm{mm}$. In Majority of patients there was no shortening. One patient needed a reoperation to reintroduce a longer hip screw as the hip screw had backed out. Following this patient had complete union and good function. Average duration of surgery was 1 hour 45 minutes. For the first 10 cases average duration of surgery was 2 hours 40 minutes and for the next 31 cases it was 87 minutes.

Technical difficulties faced during the procedure included, guide wire break in one patient, loss of reduction with varus malalignment and intertrochanteric translation (described in detail below) in 4 patients, widening of intertrochanteric area in 3 patients, difficulty in closed reduction requiring open reduction in 2 patients, fracture extension into subtrochanteric area in one patient, and trochanteric communition as a result of surgery in 2 patients. We made an attempt to categorize these technical problems and are discussed below in detail during discussion. Immediate post operative radiograph was as- sessed for hip screw position, 36 screws were in the centre or inferior position while 2 were in the superior part of neck on Antero Posterior projection. Fracture reduction was good in 34 patients, while in 4 patients we noticed translation of head and neck fragment downwards along the axis of intertrochanteric line (intertrochanteric translation), although neck shaft angle was above 130 degrees. There were 4 complications, fracture extension to subtrochanteric area in one patient, screw missing the nail in one patient, wound dehiscence in one patient, and in two cases there was backing out of lag screw (in one patient screw was reintroduced with a larger hip screw, while in other patient, fracture united with varus collapse).

We compared the results of stable fractures (type 1 and type 2) with unstable fractures (type 3 to 5) (Table 1). Mean shortening was $1.2 \mathrm{~mm}$ in stable fractures while it was $2.6 \mathrm{~mm}$ in unstable fractures. Neck shaft angle was also well maintained in unstable fractures (Mean 131 degrees in unstable fractures and 133 in stable fractures) Type 3 and 5 fractures have communition at greater trochanter. We have analyzed and compared the results of these patients with other patients (Table 2). These fractures showed slightly more shortening, with mean shortening of $2.8 \mathrm{~mm}$. All these patients had good abductor power indicating that nailing in communition of trochanter do not effect the end result.

\section{Discussion}

The technical problems faced during surgery were categorized as follows:

1) Problems related to reduction and guide wire passage: Closed reduction was successful in all except 3 patients. Good reduction was achieved after placing these patients on fracture table with boot traction. Reduction should not be problem in all fresh fractures except one or two special circumstances.

a) Long spiral fracture with subtrochanteric extension:

Table 1. Comparing outcome measures in stable fractures and unstable fractures.

\begin{tabular}{lll}
\hline Parameter & Stable fracture & Unstable fracture \\
\hline No, of patients & 16 & 25 \\
Union status & United & United \\
Available for follow up & 16 (one telephonic) & 22 (3 expired, 4 telephonic) \\
Walking status & Unaided: 12, single stick: 3, & Unaided: 8 , single stick: 12, walker assistance: 1, \\
nverage difference in harris hip score & 4 & 8 \\
Mean shortening & 1.2 mm (n $=15)$ & 2.58 mm ( $\mathrm{n}=18)$ \\
Mean neck shaft angle & 133 degrees $(\mathrm{n}=15)$ & 131 degrees $(\mathrm{n}=18)$ \\
Abductor power & Grade 4 plus in all & Grade 4 plus in 20, in 2 patients could not be assessed \\
\hline
\end{tabular}


Table 2. Comparing results of fractures with trochanteric communition and without communition.

\begin{tabular}{|c|c|c|}
\hline Parameters & Type 3 and 5 fractures & All other fractures \\
\hline Number of patients & 12 & 26 \\
\hline Neck shaft angle & 130 degrees $(n=11)$ & 133 degrees $(n=22)$ \\
\hline Mean shortening. & $2.8 \mathrm{~mm}(\mathrm{n}=11)$ & $1.63 \mathrm{~mm}(\mathrm{n}=22)$ \\
\hline Walking status & $\begin{array}{l}\text { Unaided: } 5 \text {, single stick: } 5 \text {, walker assistance: } 1 \text {, } \\
\text { not walking: } 1\end{array}$ & $\begin{array}{l}\text { Unaided: } 15 \text {, single stick: } 10 \text {, } \\
\text { walker assistance: } 1\end{array}$ \\
\hline Abductor power & Grade 4 plus in all except one & Grade 4 plus in all except one \\
\hline Union status & All united & All united \\
\hline Mean harris score difference & 7 & 6 \\
\hline
\end{tabular}

In these fractures abduction of trochanteric fragment is a problem. It is best to make a limited access at the apex of spiral fragment and put a bone holding forceps after securing reduction (Figure 1).

b) Gross communition with 4 part fracture and separation of trochanteric fragment. In these fractures posterior sagging of the trochanteric fragment is a problem [22]. Due to this, there will be excessive anteversion of neck leading to difficulty in passing the nail as well as subsequent hip screw placement. At the time of passing nail, an assistant is asked to push the trochanteric fracture up with one hand and then press the distal fragment down from the other hand. One should also observe the passage of nail into distal fragment carefully with image, so by manipulating jig, nail can be passed into distal fragment. There are also reports of passing a pin into the proximal fragment into acetabulum so that distal fragment can be moved independent of proximal fragment, although we have not tried this method [23].

Guide wire insertion can be problematic at times. Standard entry point for PFN is tip of greater trochanter. However in intertrochanteric fractures fracture involves tip of trochanter with communition. If it is a simple fracture extending to tip of trochanter without communition, it is easy to put guide pin as fracture site itself provides the entry point. But in practice, there is always some communition at tip of trochanter or fracture line is not exactly through tip. In fact we have seen fracture extending to $3-4 \mathrm{~mm}$ lateral to tip of trochanter (Figure
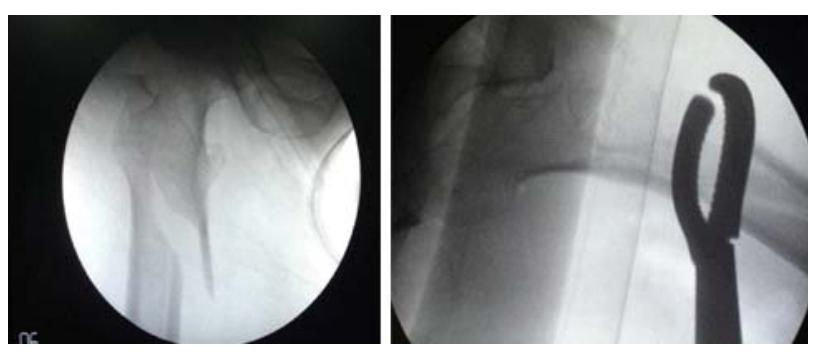

Figure 1. Long spiral fracture reduced by bone holding forceps.
2). Due to this even if an entry is made in tip of trochanter, due to narrow bone bridge lateral to tip of trochanter and medial to fracture line, guide wire and subsequent reamers fall into fracture line thus making the entry lateral to tip of trochanter. Some times there will be communition with an additional coronal split so that there is no lateral support at the entry region while reaming or putting nail. All these things lead to lateral entry of nail. However we believe these things will not affect the final outcome as communited fragments sit around the nail and mould and unite thus there will not be significant abductor weakness. Important technical aspect here is to start the entry from tip of trochanter and slightly anterior in the lateral plane and then aim to pass the guide wire into shaft of femur in the centre, once guide wire is passed into shaft we ignore the lateralization of guide wire at tip of trochanter. Edward T. Su et al. [24] found that entry of either pyriformis fossa or tip of greater trochanter do not make any difference in AP projection. During subsequent reaming and passing nail, we take care to adduct limb nicely and also keep the reamer or nail handle as close to flank as possible so that reaming and nail passes as medial as possible (reaming of lateral edge of proximal fragment). If the guide wire goes out

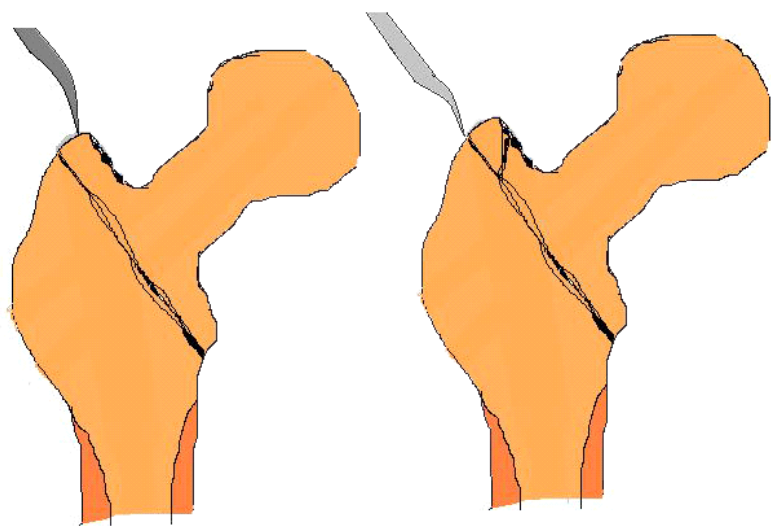

Figure 2. Shows how entry awl can slip from tip of trochanter into fracture site. The guide wire also takes the same route. 
through the medial cortex, cannulated reamer is passed over the guide wire and by manipulating the reamer, towards the centre of medullary cavity, guide wire can be directed easily in to the medullary canal (Figure 3).

2) Problems while passing nail: Two important problems were noticed during nail insertion. One is possibility of fracture of proximal femur, second one is related to loss of reduction. During passage of nail, it can breach medial or anterior cortex, and is responsible for proximal femur fracture while passing the nail. This happens just below lesser trochanter. If one is not able to identify the problem, then one can end up with subtrochanteric or proximal femoral fracture (Figure 4). In our series, one patient developed subtrochanteric fracture, but then we ignored the fracture and passed the nail into distal fragment, and union of both intertrochanteric and iatrogenic fractures were achieved. In literature, there are reports of such fractures due to failure to anticipate this problem.

Technical trips for easy passage of nail and prevention of proximal femoral fracture:

a) Always ream adequately. Our surgical technique included reaming the medullary cavity initially upto 10 $\mathrm{mm}$ beyond isthmus if there is narrowing of proximal femoral canal with adequate good cortical bone stock. In very elderly with gross osteoporosis with wide canal this step can be bypassed. Then we ream upto $15 \mathrm{~mm}$ upto the level of lesser trochanter. This will allow passage of nail easily.

b) Pass a smaller diameter nail which goes easily. Any-

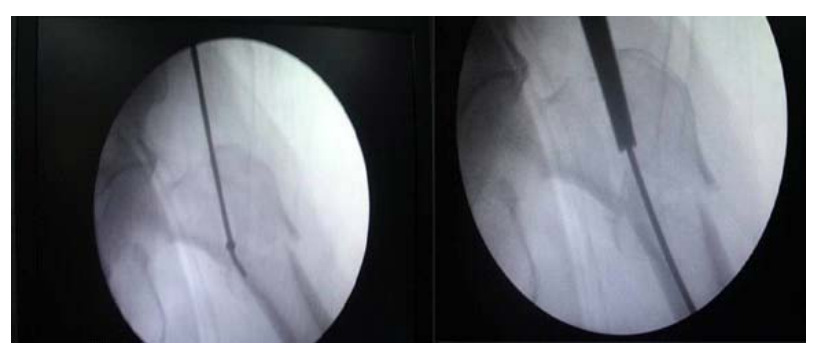

Figure 3. Use of cannulated awl to guide the guide wire.

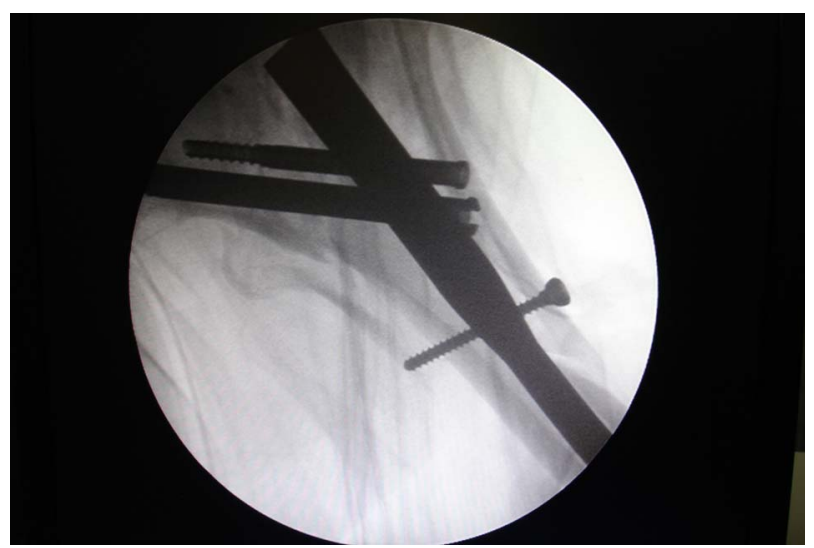

Figure 4. shows iatrogenic proximal femoral fracture. way, unless there is subtrochanteric extension, diameter of distal part of nail is unlikely to influence stability of fixation of fracture. The proximal diameter of nail is same in all nails.

c) While passing the nail, continuously monitor with C-arm and identify the path of nail and ensure it does not break either anterior cortex or medial cortex. Then the direction of nail can be adjusted. A slightly anterior entry point in the lateral projection could prevent hitting the anterior cortex while adducting the limb and keeping the nail insertion device handle as close to flank as possible will help to prevent breach of medial cortex.

We also noticed another important phenomena during nail insertion, as the broader portion of nail passes through the intertrochanteric area, the proximal fragment translates along the intertrochanteric line. This is a problem not discussed by any of earlier authors although varus reduction due to entry reamer was discussed by Hak D. J. et al. [25]. As nail takes its position in the proximal half of femur, the proximal end of nail pushes proximal fragment downwards (Figures 5, 6(a) and (b)). Usually at this position, the tip of nail will be hitting against medial cortex and manipulating jig medially to direct the nail to central part of canal will produce lever action and larger proximal part of nail pushes the proximal fragment downwards along with some distraction at fracture site. We call this translation in the plane of intertrochanteric line and can be associated with varus reduction also. Also the distal fragment is pushed by the nail laterally causing widening at intertrochanteric area (distraction). This phenomenon is unlikely to effect the final result as long as neck shaft angle is maintained and fracture has not aligned into a varus position. We had persistent translation in two cases but union and good functional result was achieved in both these patients. However the distraction effects of nail can be minimized by over reaming the

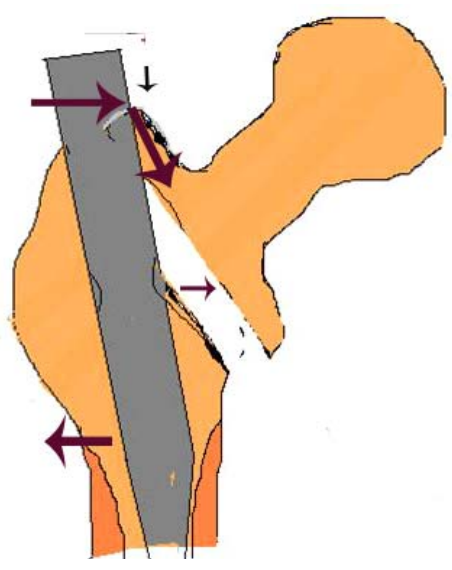

Figure 5. Mechanism of intertrochanteric translation and displacement. Big arrows show deforming force exerted by the nail on the bone, while small arrows show direction of fracture displacement. 
proximal part of femur till subtrochanteric area upto 15 $\mathrm{mm}$ with flexible reamers. The solid reamers can push trochanter laterally without actually reaming it. Recently, Hak D. J. et al. [25] mention about overdistraction while entry reamer, this will ream the lateral edge of medial fragment, which is the main reason for varus and distraction, but we have found our methods useful. Also one can insert a long artery forceps with a small incision on the anterolateral aspect of thigh in the lesser trochanter region under the neck of femur and push it up while passing nail preventing translation (Figure 7). Excessive reaming also prevents distraction as nail goes easily, an assistant can maintain some pressure over trochanter while passing last part of nail. There are also reports of removing a small bone from lateral edge of medial fragment [26]. Even after this if some distraction is present this can be corrected by using the lag screw first to compress the fracture site. One should study the neck shaft

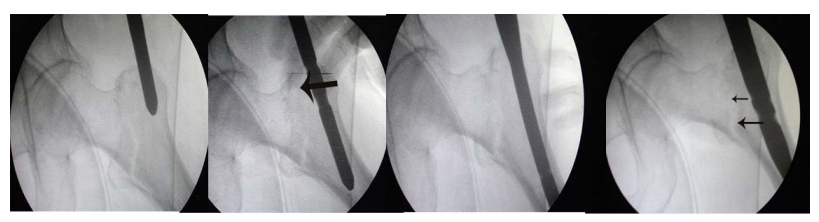

(a)

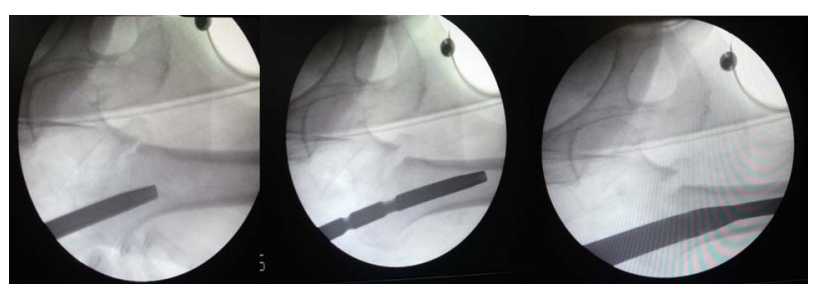

(b)

Figure 6. (a) and (b) Shows fracture displacement as nail passes through proximal femur.

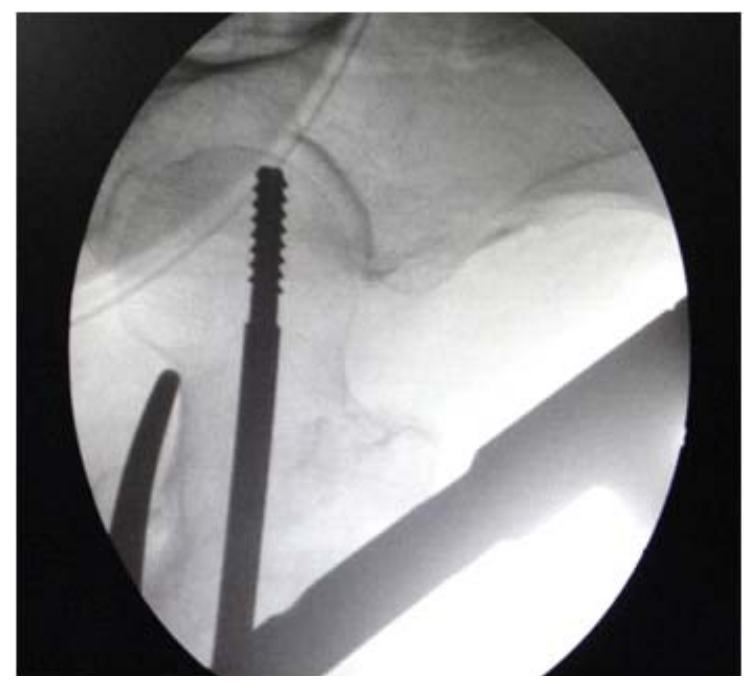

Figure 7. Use of artery forceps to reduce the intertrochanteric translation and distraction. angle clearly during this malreduction, and if there is varus, then that should be corrected as it is impossible to place 2 screws correctly, while a little bit of translation or distraction can be accepted.

3) Problems while locking the nail: One should always pass the lag screw in the inferior part of neck in AP projection and centre in the lateral plane. If lag screw is in the centre then sometimes derotation screw goes too superiorly and even out of superior cortex of neck of femur. In two of our cases, position of lag screw was slightly superior and in both cases screw had backed out.

Initially we were using derotation screw first and then the compression screw, but when there was some widening of fracture, we found it would be advantageous to put the compression screw first. We usually put both guide wires first, then put lag screw to compress the fracture keeping derotation screw guide wire in place and then finally place the derotation screw. One can put the derotation screw first, but then achieving compression across fracture site can be a difficult task. In literature there is no clear consensus on this.

There can be problems while reaming over guide wire for hip screw, as guide wire may bend slightly as it reaches subchondral bone (Figure 8). Now if one starts reaming over this, guide wire may break. We had 2 such incidences, in one case we removed the guide wire, in other patient, we could not. Also in 2 cases we were able to detect bending of guide wire, we could remove the guide wire before it actually broke. To prevent guide wire breakage, we reamed upto subchondral level, then pulled the guide wire upto subchondral level and then proceeded with reaming under $\mathrm{C}$-arm guidance. The guide wire was reinserted after the removal of reamer for screw insertion. The guide wire can bend at outer cortex also but this can be prevented with predrilling the entry point at lateral cortex with a drill bit. Some times there will be problems with negotiating the reamer for hipscrew through the nail due to some manufacture or implant assembly related problems. We had two such problems, one for hip screw and other for derotation screw. Reaming with smaller sized reamer and proceeding with regular reamer will help. One should also check the jig whether it is loosened or not.

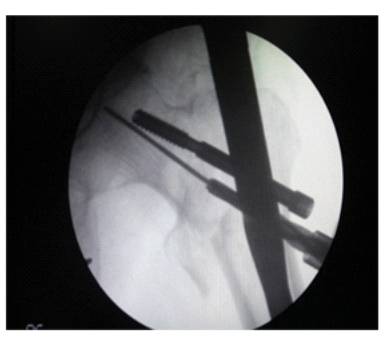

(a)

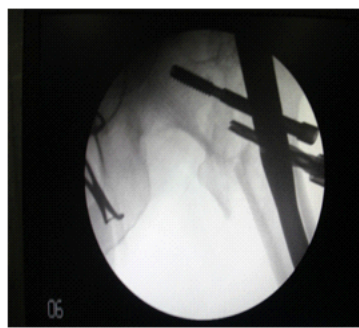

(b)
Figure 8. Showing (a) bending of guide wire and (b) subsequent reaming after removal of guide wire. 
In literature, when we analyse failures of PFN, we found that most of these are due to faulty techniques, like failure to reduce properly, surgery done by residents, failure to anticipate nail touching anterior cortex while hammering, use of larger decortical screw. J. Pajarinen et al. [4] in their study of comparison between dynamic hip screw and proximal femoral nail found that use of PFN has a positive effect on the speed of restoration of walking mainly due to restoration of near normal anatomy. Al-Yassari et al. [27] and Simmermacher et al. [28] also observed restoration of preoperative mobility in approximately $40 \%-50 \%$ of the patients treated with PFN. Pajarinen explains that early walking ability in PFN is due to better anatomical restoration in comparison to DHS where there is greater impaction of fracture leading to shortening of femoral neck. M. S. G. Ballal et al. [9] in their study found $5 \%$ PFN failures and opined that In PFN fixations, proper alignment between the 2 main fragments and proper placement of the lag screws in the femoral head should be ensured. In their study it so happened that all failed PFN fixations were performed by registrars.

Our study concurs with these findings, as mean shortening in our series was less than $4 \mathrm{~mm}$. Only one patient had a shortening of $2.5 \mathrm{~cm}$. This is definitely less than shortening observed with DHS (mean shortening 10.8 $\mathrm{mm}$ in J. Pajarinen et al.). However, J. Pajarinen et al. [4] do not establish clear superiority of PFN in view of increased operation time, same redisplacement rate and final outcome at one year being same. Similarly in another study by Saudan et al. [3], they found no statistical difference between two groups with reduced mobility in PFN group and higher screw failure rate for PFN. However it was mentioned that in these screw failure groups, the screws were placed more superiorly. Looking at these studies, we believe it is more of technical failure rather than failure of implant, if properly done we believe PFN gives superior results. We believe during reduction of these fractures accurate anatomical reduction is not required as it is impossible in unstable fractures, but reduce to a position where the neck shaft angle is maintained and there is medial continuity. One should not do varus reduction. If correct neck shaft angle and version are maintained, irrespective of communition, irrespective of nonanatomical reduction fracture will unite with minimal shortening. Placement of hip screw is critical to the final result as placement of hip screw in superior part of neck can lead to cut out or varus collapse. In view of this, we believe, most important technical aspect of this surgery is maintaining the proper neck shaft angle and placing the hip screw in the centre of hip. Both are interlinked as screw placement angle is prefixed and hence unless good neck shaft angle is achieved, it is impossible to put the hip screw correctly. In the AP Projection, varus angula- tion or intertrochanteric translation is the main problem to place the screw in centre, which is largely due to broad proximal part of nail pushing the lateral edge of proximal fragment down as described earlier in our analysis of technical results and supported by others (Huk D. J. et al. [25]), while in lateral projection posterior sagging of trochanter is the problem in putting the hip screw centrally.

T. Moihara et al. [14] also opine that hip pin should be shorter by atleast $15 \mathrm{~mm}$ otherwise it could take weight and can back out or migrate into joint leading to cut out. In our study, we placed hip pin of at least $20 \mathrm{~mm}$ shorter compared to hip screw. As a result we had only one loosening of lag screw and one patient had slight varus collapse (Figure 9). This is in contrast to some of earlier reports $[3,27,28]$ which give higher cut out rates.

In our series, our mean neck shaft angle was 132 degrees and mean shortening was just $2 \mathrm{~mm}$, which shows excellent results compared to any of previous DHS results, We have also shown that the results are excellent when there is gross communition including loss of medial and lateral walls (Figures $\mathbf{1 0}$ and $\mathbf{1 1}$ show type 5 fractures with good union). Table number 2 shows excellent maintenance of neck shaft angle and union in such patients, and also good Harris hips score, although, our comparison with preinjury scores may not be reliable completely, as preinjury scores are very subjective and retrospective. But good union and good walking ability

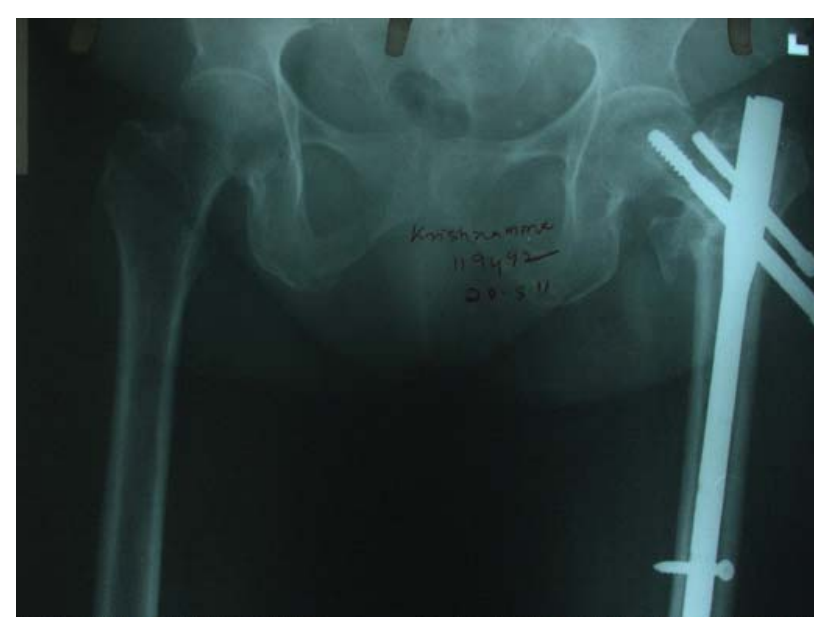

Figure 9. Back out of lag screw with varus malunion.

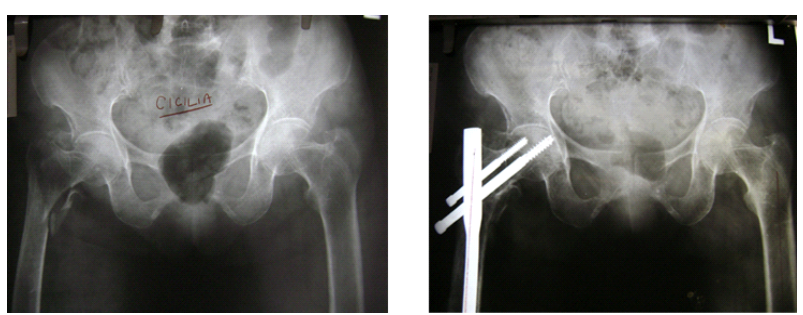

Figure 10. A type 5 fracture with good outcome. 
indicated that the varus collapse seen with DHS is less likely in these patients. By giving importance to technical problems mentioned above it is possible to avoid most of technical problems mentioned in literature, while for people who are already experienced in placing interlocking nails for complex femoral fractures, it is not that difficult to master the technique and reduce the time for surgery also. Once the proper technique is mastered, operation time, bleeding and risk of infection are minimal with Proximal femoral nail.

Communition of greater trochanter: Communition of greater trochanter leads to technical difficulty in passing nail and nail entry point may get lateralized, and tip of trochanter can get splayed. However in the final followup, almost all patients have good abductor strength and do not have any significant disability related to that (Figures 12 (a)) and (b)) show final result following nailing

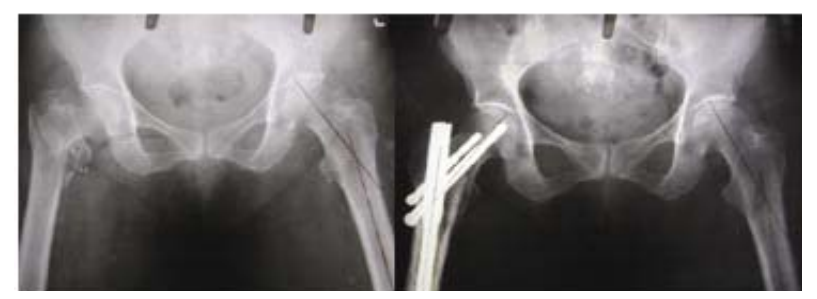

Figure 11. Another case of type 5 fracture with good union.

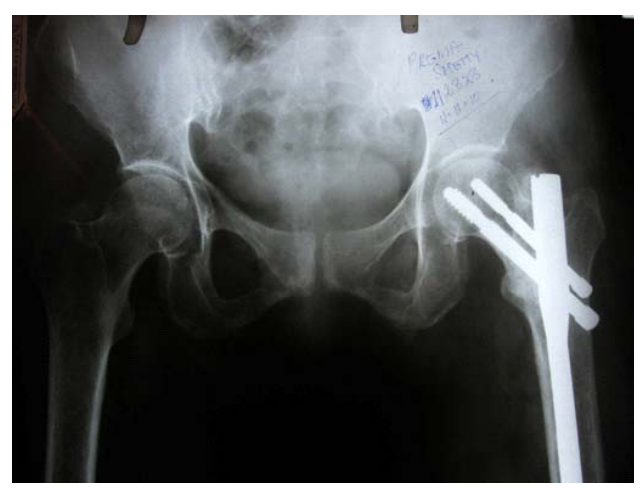

(a)

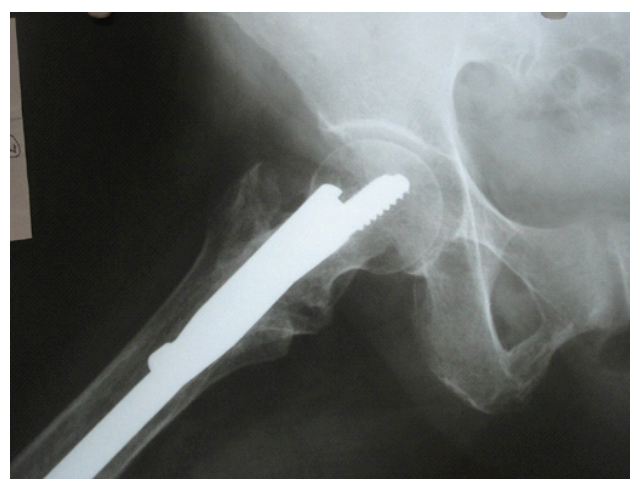

(b)

Figure 12. (a) and (b) Type 3 fracture with gross trochanteric communition showing union. in gross trochanteric communition).

\section{Conclusion}

Our study shows that, proximal femoral nailing produces good results with negligible varus collapse and shortening in unstable fractures and hence should be preferred in these fractures. Although surgery is technically required, if one gives careful attention to minute technical details at each step as discussed above, good outcome can be achieved and this should not be a problem for those surgeons who are already experienced in nailing complex femoral shaft fractures. In the final analysis, we believe, achieving a good reduction between two main fragments without varus malalignment and placement of hip screw in a correct position are two important technical aspects which prevent most of the complications associated with these procedures.

\section{REFERENCES}

[1] R. Marks, "Hip Fracture Epidemiological Trends, Outcomes, and Risk Factors, 1970-2009," International Journal of General Medicine, Vol. 3, 2010, pp. 1-17.

[2] K. P. Chang, J. R. Center, T. V. Nguyen and J. A. Eisman, "Incidence of Hip and Other Osteoporotic Fractures in Elderly Men and Women: Dubbo Osteoporosis Epidemiology Study," Journal of Bone and Mineral Research, Vol. 19, No. 4, 2004, pp. 532-536. doi:10.1359/JBMR.040109

[3] M. Saudan, A. Lubbeke, C. Sadowskil, N. Riand, R. Stern and P. Hoffmeyer, "Pertrochanteric Fractures: Is There an Advantage to an Intramedullary Nail? A Randomized Prospective Study of 206 Patients Comparing the Dynamic Hip Screw and Proximal Femoral Nail," Journal of Orthopaedic Trauma, Vol. 16, No. 6, 2002, pp. 386-393.

[4] J. Pajarinen, J. Lindahl, O. Michelsson, V. Savolainen and E. Hirvensalo, "Pertrochanteric Femoral Fractures Treated with a Dynamic Hip Screw or a Proximal Femoral Nail. A Randomized Study Comparing Post-Operative Rehabilitation," Journal of Bone and Joint Surgery, Vol. 87, No. 1, 2005, pp. 76-81.

[5] M. R. Baumgaertner, S. L. Curtin and D. M. Lindskog, "Intramedullary versus Extramedullary Fixation for the Treatment of Intertrochanteric Hip Fractures," Clinical Orthopaedics and Related Research, Vol. 348, 1998, pp. 87-94.

[6] T. R. C. Davis, J. L. Sher, A. Horsman, M. Simpson, B. B. Porter and R. G. Checketts, "Intertrochanteric Femoral Fractures, Mechanical Failure after Internal Fixation," Journal of Bone and Joint Surgery, Vol. 72, No. 1, 1990, pp. 26-31.

[7] C. I. Adams, C. M. Robinson, C. M. Court-Brown and M. M. Mcqueen, "Prospective Randomized Controlled Trail of an Intramedullary Nail versus Dynamic Screw and Plate for Intertrochanteric Fractures of the Femur," Journal of Orthopaedic Trauma, Vol. 15, No. 6, 2001, pp. 394-400. doi:10.1097/00005131-200108000-00003 
[8] W. M. Gadegone and Y. S. Salphale, "International Orthopaedics, Proximal Femoral Nail-An Analysis of 100 Cases of Proximal Femoral Fractures with an Average Follow up of 1 Year," International Orthopaedics, Vol. 31, No. 3, 2007, pp. 403-408.

[9] M. S. G. Ballal, N. Emms, M. Ramakrishnan and G. Thomas, "Proximal Femoral Nail Failures in Extracapsular Fractures of the Hip," Journal of Orthopaedic Surgery, Vol. 16, No. 2, 2008, pp. 146-149.

[10] L. A. Flores, I. J. Harrington and M. Heller, "The Stability of Intertrochanteric Fractures Treated with a Sliding Screw-Plate," Journal of Bone and Joint Surgery, Vol. 72, No. 1, 1990, pp. 37-40.

[11] S. H. Bridle, A. A. A. D. Patel, M. Bircher, et al., "Fixation of Intertrochanteric Fractures of the Femur: A Randomized Prospective Comparison of the Gamma Nail and Dynamic Hip Screw," Journal of Bone and Joint Surgery, Vol. 73, No. 2, 1991, pp. 330-334.

[12] S. C. Halder, "The Gamma Nail for Peritrochanteric Fractures," Journal of Bone and Joint Surgery, Vol. 74, No. 3, 1992, pp. 340-344.

[13] I. B. Schipper, E. W. Steyerberg, R. M. Castelein, F. H. W. M. van der Heijden, P. T. den Hoed, A. J. H. Kerver and A. B. van Vugt, "Treatment of Unstable Trochanteric Fractures, Randomised Comparison of the Gamma Nail and the Proximal Femoral Nail," Journal of Bone and Joint Surgery, Vol. 86, No. 1, 2004, pp. 86-94.

[14] T. Morihara, S. Tokugawa, S. Fujita, K. Chatani and T. Kubo, "Proximal Femoral Nail for Treatment of Trochanteric Femoral Fractures," Journal of Orthopaedic Surgery, Vol. 15, No. 3, 2007, pp. 273-277.

[15] W. M. Gadegone and Y. S. Salphale, "Short Proximal Femoral Nail Fixation for Trochanteric Fractures," Journal of Orthopaedic Surgery, Vol. 18, No. 1, 2010, pp. 3944.

[16] M. Uzun, E. Ertürer, İ. Öztürk, Ș. Akman, F. Seçkin and İ. B. Özçelik, "Long-Term Radiographic Complications Following Treatment of Unstable Intertrochanteric Femoral Fractures with the Proximal Femoral Nail and Effects on Functional Results," Acta Orthop Traumatol Turc, Vol. 43, No. 6, 2009, pp. 457-463.

[17] E. N. Kubiak, M. Bong, S. S. Park, F. Kummer, K. Egol and K. J. Koval, "Intramedullary Fixation of Unstable Intertrochanteric Hip Fractures, One or Two Lag Screws," Journal of Orthopaedic Trauma, Vol. 18, No. 1, 2004, pp. 12-17. doi:10.1097/00005131-200401000-00003

[18] M. J. Parker and H. H. G. Handoll, "Gamma and Other Cephalocondylic Intramedullary Nails versus Extramedul- lary Implants for Extracapsular Hip Fractures in Adults," Cochrane Database of Systematic Reviews, No. 3, 2008, Article ID: CD000093.

[19] H. Pervez, M. J. Parker, G. A. Pryor and L. Lutchman, "Classification of Trochanteric Fracture of the Proximal Femur: A Study of the Reliability of Current Systems; Nishan Chirodian Injury," Injury, Vol. 33, No. 8, 2002, pp. 713-715. doi:10.1016/S0020-1383(02)00089-X

[20] E. M. Evans, "The Treatment of Trochanteric Fractures of the Femur," Journal of Bone and Joint Surgery, Vol. 31-B, 1949, pp. 190-203.

[21] W. H. Harris, "Traumatic Arthritis of the Hip after Dislocation and Acetabular Fractures: Treatment by Mold Arthroplasty. An End Result Study Using a New Method of Result Evaluation," Journal of Bone and Joint Surgery, Vol. 51, No. 4, 1969, pp. 737-755.

[22] J. Langford and A. Burgess, "Nailing of Proximal and Distal Fractures of the Femur: Limitations and Techniques," Journal of Orthopaedic Trauma, Vol. 23, No. 5, 2009, pp. S22-S25. doi:10.1097/BOT.0b013e31819f2797

[23] D. M. Lindskog and M. R. Baumgaertner, "Unstable Intertrochanteric Hip Fractures in the Elderly," Journal of the American Academy of Orthopaedic Surgeons, Vol. 12, No. 3, 2004, pp. 179-190.

[24] E. T. Su, H. DeWal, R. Sanders, F. J. Kummer, M. Mujtaba and K. J. Koval, "Effect of Piriformis versus Trochanteric Starting Point on Fixation Stability of Short Intramedullary Reconstruction Nails," Bulletin Hospital for Joint Diseases, Vol. 60, No. 2, 2001, pp. 67-71.

[25] D. J. Hak and C. Bilat, "Avoiding varus Malreduction during Cephalomedullary Nailing of Intertrochanteric Hip Fractures," Archives of Orthopaedic and Trauma Surgery, Vol. 131, No. 5, 2011, pp. 709-710.

[26] J. T. Riehl and J. C. Widmaier, "Techniques of Obtaining and Maintaining Reduction during Nailing of Femur Fractures," Orthopedics, Vol. 32, No. 8, 2009, p. 581. doi:10.3928/01477447-20090624-17

[27] G. Al-Yassari, R. J. Langstaff, J. W. M. Jones and M. Al-Lami, "The AO/ASIF Proximal Femoral Nail for the Treatment of Unstable Trochanteric Femoral Fracture," Injury, Vol. 33, No. 5, 2002, pp. 395-399. doi:10.1016/S0020-1383(02)00008-6

[28] R. K. J. Simmermacher, A. M. Bosch and C. Ven der Werken, "The AO/ASIF Proximal Femoral Nail, a New Device for the Treatment of Unstable Proximal Femoral Fractures," Injury, Vol. 30, No. 5, 1999, pp. 327-332. doi:10.1016/S0020-1383(99)00091-1 